\title{
COMPROMISOS DE DESEMPEÑO, INCENTIVOS Y EVALUACIÓN DEL TRABAJO ACADÉMICO EN UNIVERSIDADES CHILENAS*
}

\section{Introducción}

El presente artículo aborda dos temas que han comenzado a tomar una creciente importancia en la gestión universitaria chilena: los compromisos de desempeño y los incentivos al trabajo académico.

En algunas universidades, ambos tienen ya una tradición de más de diez años. El origen de su utilización está asociado a los procesos de planificación estratégica iniciados a fines de los años 90, cuando el Programa de Mejoramiento de la Calidad y Equidad de la Educación Superior (MECESUP) los planteó como requisito para acceder al Fondo Concursable.

Los compromisos de desempeño y los incentivos al trabajo académico son instrumentos operativos de la planificación estratégica y ambos son complementarios. Sin embargo, para su funcionamiento óptimo requieren de un tercer procedimiento: la evaluación. Este artículo sólo enuncia este tercer punto y se focaliza más detalladamente en los dos primeros.

Tanto los compromisos de desempeño como los incentivos al trabajo académico han sido implementados en las universidades chilenas de manera muy diversa. Por lo mismo, y debido a la falta de estudios exhaustivos que analicen este tema, las líneas que siguen lo abordan desde una perspectiva más conceptual que empírica. No obstante, en algunos casos se presentan ejemplos concretos. Incluso,

* Este artículo fue elaborado a partir del Proyecto Anillos 'Programa de Investigación en Políticas de Educación Superior', financiado por CONICYT (Proyecto SOC01, adjudicación: marzo de 2008). 
cuando parece necesario, se incorporan modelos y fórmulas específicas de algunas universidades.

Las principales fuentes de información de este artículo fueron una serie de entrevistas realizadas durante 2003 a miembros de los equipos directivos de la Pontifica Universidad Católica de Valparaíso, de la Pontificia Universidad Católica de Chile y de la Universidad de Talca; reglamentos y formularios recopilados en igual periodo ${ }^{1}$, y las presentaciones del Taller de Gestión en Educación Superior, organizado en noviembre de 2007 por la Universidad de Talca, la Universidad de La Frontera y la Universidad de Ciencias Aplicadas de Osnabrück, Alemania.

A pesar del detallado tratamiento que por momentos se hace del tema, el objetivo de este artículo no es señalar que la implementación de compromisos de desempeño y de incentivos al trabajo académico es la única forma de gestionar una universidad. Menos aún afirmar que determinadas modalidades de compromisos y estructuras de incentivos son mejores que otras. Al contrario, lo que se busca es sólo mostrar una de las opciones de gestión y dar cuenta de la variedad y flexibilidad que ésta puede asumir. Además, persigue discutir algunas de sus complejidades.

\section{Niveles}

Los compromisos de desempeño se utilizan, en general, en tres niveles:

- Gubernamental: consiste en establecer un contrato entre el gobierno y una institución, con el objeto de negociar ciertos financiamientos basales que entregará el Estado contra determinados indicadores de desempeño que la universidad respectiva deberá alcanzar. Esta modalidad es utilizada, por ejemplo, entre el Estado y sus universidades en Francia o entre los Estados federales alemanes y las universidades públicas. En Chile también se ha implementado

1 Estas entrevistas e instrumentos se realizaron y recopilaron en un trabajo realizado en la Universidad Católica de la Santísima Concepción, con el objeto de redefinir las políticas de trabajo académico. 
recientemente este tipo de contratos bajo el nombre de "Convenios de Desempeño" 2 .

- Institucional: en este caso, los compromisos se contraen entre la dirección central de una universidad y sus unidades académicas. Éstas comprometen determinadas actividades y logros (en general anuales) ante la "Casa Central". Del grado de éxito en el cumplimiento de los objetivos dependerá, en gran parte, su capacidad de negociación para construir su plan de desarrollo y su presupuesto anual ${ }^{3}$.

- Individual: corresponde a los compromisos que cada profesor contrae con su unidad académica y que, idealmente, deben tributar al realizado entre ésta y la administración central de la universidad.

Las líneas que siguen se focalizan en este tercer nivel.

\section{III. Ámbitos del desempeño académico y flexibilidad}

a) Ámbitos de desempeño

Los compromisos de desempeño contemplan las áreas que se reconocen como tradicionales en la función académica: docencia, investigación, extensión y administración. Es decir, cada profesor contrae con su unidad académica o institución determinadas obligaciones de trabajo y productividad anuales.

Recientemente se ha evidenciado que el surgimiento de otras funciones institucionales y de nuevas demandas externas requiere de una actualización de la estructura de los instrumentos

2 Durante 2007 se implementaron cuatro convenios de desempeño entre el Estado y las universidades de Tarapacá, de Chile, del Bío Bío y de La Frontera, por un monto total superior a los doce mil quinientos millones de pesos, considerando las contrapartes institucionales, que sumaron más de dos mil quinientos millones (www.mecesup.cl). En 2008 se pondrá en marcha un nuevo convenio de desempeño entre el Estado y la Universidad de Chile por un monto de 50 millones de dólares, exclusivamente en el ámbito de las Ciencias Sociales y Humanidades.

3 En este nivel es posible, dependiendo del tamaño de la organización, encontrar otra desagregación entre las facultades y sus departamentos o centros (Ziegele, 2007a). 
y algunas universidades han incorporado ya dimensiones como "perfeccionamiento" y "transferencia tecnológica".

Al interior de los ámbitos del desempeño académico se realiza una serie de desagregaciones en actividades. El siguiente ejemplo ha sido elaborado a partir de compromisos utilizados en diversas universidades (diagrama 1):

Diagrama 1. Ámbitos del desempeño académico

\begin{tabular}{|c|c|c|c|}
\hline \multicolumn{4}{|c|}{ Ámbitos del desempeño (tradicionales) } \\
\hline & & & \\
\hline $\begin{array}{l}\text { DOCENCIA } \\
\text { - } \text { Cursos } \\
\text { - Atención de } \\
\text { alumnos } \\
\text { - Elaboración de } \\
\text { material docente } \\
\text { - Dirección de tesis } \\
\text { - Otras } \\
\end{array}$ & $\begin{array}{l}\text { INVESTIGACIÓN } \\
\text { - Publicación de } \\
\text { artículos } \\
\text { - Ejecución de } \\
\text { proyectos de } \\
\text { investigación } \\
\text { - Formulación de } \\
\text { proyectos }\end{array}$ & $\begin{array}{l}\text { EXTENSIÓN } \\
\text { - Conferencias } \\
\text { abiertas al público } \\
\text { - Charlas y visitas a } \\
\text { colegios } \\
\text { - Artículos de prensa } \\
\text { - Otros } \\
\end{array}$ & $\begin{array}{l}\text { ADMINISTRACIÓN } \\
\text { - Gestión directa } \\
\text { superior } \\
\text { - Dirección de } \\
\text { programas o } \\
\text { departamentos } \\
\text { - Dirección de } \\
\text { proyectos de }\end{array}$ \\
\hline & $\begin{array}{l}\text { - Estadías de } \\
\text { investigación } \\
\text { - Presentación } \\
\text { de trabajos en } \\
\text { congresos de la } \\
\text { especialidad } \\
\text { - Otros }\end{array}$ & & $\begin{array}{l}\text { desarrollo } \\
\text { - Organización } \\
\text { de actividades } \\
\text { académicas } \\
\text { - Otros } \\
\end{array}$ \\
\hline
\end{tabular}

\begin{tabular}{|l|l|}
\hline \multicolumn{2}{|c|}{ Ámbitos del desempeño (nuevos) } \\
\cline { 2 - 2 } \multicolumn{1}{|c|}{} & \\
\hline PERFECCIONAMIENTO & TRANSFERENCIA \\
- Estudios de & TECNOLÓGICA \\
posgrado (magíster o & - Asesorías y \\
doctorado) & consultorías \\
- Estudios de & Evaluación de \\
posdoctorado & proyectos de \\
- Otros & desarrollo externos \\
\hline
\end{tabular}

b) Flexibilidad

Los compromisos de desempeño ponderan de distinta manera y otorgan diversos grados de flexibilidad a cada uno de los ámbitos definidos. Ambas situaciones se derivan del ajuste que se intenta 
hacer con las políticas y normas de trabajo académico vigentes en cada institución.

En general, el ámbito en que existe menos flexibilidad es la docencia. Es decir, se deja muy poco espacio a la negociación del volumen o tiempo de trabajo dedicado a ella (no así a las tareas asociadas). Esto se explica básicamente porque es concebida como una función central de las universidades y se espera que todo académico la realice, y porque es la actividad que mayor presión presupuestaria puede generar si los docentes de planta la delegan en profesores contratados por hora.

La forma de asignar el trabajo docente varía de una institución a otra. Algunas universidades especifican un número mínimo de cursos que un profesor debe dictar o de alumnos que debe atender semestralmente, otras establecen las horas de clases presenciales que se deben realizar cada semana y otras fijan porcentajes de la jornada laboral ${ }^{4}$.

Un ejemplo interesante de asignación de trabajo docente es el modelo utilizado por la Facultad de Ciencias de la Pontificia Universidad Católica de Valparaíso y que, con algunas variaciones, se implementó en la Universidad Católica de la Santísima Concepción en 2004. La primera discriminaba por tipo de contrato para la asignación del número mínimo de horas de docencia presencial directa; la segunda asoció, además, la jerarquía académica (tabla 1):

4 Para ello utilizan fórmulas que integran la docencia presencial, el número de alumnos, el horario de atención de alumnos y otros factores. 
Tabla 1. Docencia directa presencial semanal según categoría y jornada

\begin{tabular}{|l|c|c|c|}
\hline Profesores con jornada entre 34 y 44 horas \\
\hline Categoría & Hrs. pedagógicas* & Módulos & Hrs. cronológicas \\
\hline Auxiliar & 14 & 7 & $9^{\prime} 20^{\prime \prime}$ \\
\hline Adjunto & 12 & 6 & $8^{\prime} 00^{\prime \prime}$ \\
\hline Titular & 10 & 5 & $6^{\prime} 40^{\prime \prime}$ \\
\hline Profesores con jornada entre 23 y 33 horas \\
\hline Auxiliar & 12 & 6 & $8^{\prime} 00^{\prime \prime}$ \\
\hline Adjunto & 10 & 5 & $6^{\prime} 40^{\prime \prime}$ \\
\hline Titular & 8 & 4 & $5^{\prime} 20^{\prime \prime}$ \\
\hline Profesores con jornada entre 12 y 22 horas & \multicolumn{1}{|l|}{} \\
\hline Auxiliar & 10 & 5 & $6^{\prime} 40^{\prime \prime}$ \\
\hline Adjunto & 8 & 4 & $5^{\prime} 20^{\prime \prime}$ \\
\hline Titular & 6 & 3 & $4^{\prime} 00^{\prime \prime}$ \\
\hline
\end{tabular}

* Hora pedagógica de 40 minutos

En general, en los ámbitos de investigación, extensión y administración los compromisos de desempeño permiten una mayor flexibilidad. No obstante, producto del concepto de universidad que predomina en Chile, existe una expectativa bastante generalizada de que todo profesor debe realizar al menos algunas tareas en el área de investigación.

Las modalidades de ponderación en estos ámbitos son similares a las ya consignadas. Es decir, los profesores pueden especificar el número de horas que dedicarán a cada tarea (hasta completar su jornada) o comprometer ciertas actividades o productos específicos.

La mayor flexibilidad en estos ámbitos se relaciona con que en los últimos años, dados los diversos talentos e intereses de los académicos, no se les puede exigir dedicaciones ni rendimientos homogéneos.

\section{Incentivos}

Como se señaló, los incentivos operan como un complemento de los compromisos de desempeño y se utilizan para orientar desarrollos 
organizacionales a partir de su focalización hacia determinadas actividades o productos.

a) Los objetivos estratégicos institucionales y la política pública

Tal como con los compromisos de desempeño, también con los incentivos se pueden privilegiar ciertos ámbitos o actividades de interés institucional. Es decir, ambos pueden ser acoplados a los objetivos de desarrollo organizacional establecidos en la planificación estratégica. De ese modo, se transforman en una herramienta para su logro, al orientar el trabajo de los académicos en la dirección que interesa a la universidad.

Algunas instituciones han alineado además los incentivos con determinadas políticas públicas, con el fin de lograr una mayor concordancia con éstas o conseguir un mejor acceso a las subvenciones asociadas. Por ejemplo, se privilegian las publicaciones indexadas en ISI (Institute for Scientific Information) o SciELO (Scientific Electronic Library Online), la adjudicación de proyectos de investigación del Concurso Regular de FONDECYT (Fondo de Desarrollo de Ciencia y Tecnología) o la obtención de grados académicos por parte de sus profesores. Esto permite, en el caso de las universidades del Consejo de Rectores, una mayor adjudicación de Aporte Fiscal Directo (AFD) ${ }^{5}$. Otras instituciones incentivan la presentación de proyectos al Fondo Concursable del MECESUP.

b) Tipos de incentivos

De manera creciente, los incentivos entregados por las universidades son monetarios. En la mayoría de los casos van directamente a los académicos en forma de bonos adicionales al sueldo, en ocasiones imponibles y tributables. Así, un porcentaje importante de éste se torna variable y depende de su propia productividad.

5 Esta estrategia explica parte del éxito de la Universidad de Talca, que ha aumentado sistemáticamente su participación en el AFD (Bernasconi y Rojas, 2004: 167). 
Su implementación, sin embargo, no ha estado exenta de controversias y ha contribuido a tensionar los distintos conceptos sobre el trabajo académico.

Dos son las posiciones principales: una señala que las funciones académicas tradicionales no han cambiado y, por lo mismo, su desarrollo está considerado dentro del contrato de trabajo y remuneración. Un pago extra por realizar lo que siempre se ha realizado contribuye, a juicio de quienes defienden esta postura, a una suerte de desnaturalización y monetarización del trabajo académico; otra afirma que es necesario premiar a quienes producen y se comprometen más con la organización, y que la mejor forma de hacerlo es a través de incentivos económicos.

Un argumento adicional a la postura a favor de los incentivos económicos surge en aquellas universidades donde los sueldos son percibidos como más bajos que en otras. En esos casos -señalan- los incentivos operarían como un complemento que, bien orientado, permitiría equiparar las remuneraciones de los más productivos a los valores reales de mercado.

También existen incentivos no monetarios al trabajo académico. Un ejemplo es la modalidad de "descarga" horaria de la Facultad de Ciencias de la Pontificia Universidad Católica de Valparaíso, también aplicada en la Universidad Católica de la Santísima Concepción en 2004 (que puede operar o no como complemento de otros incentivos económicos). En este caso, se reduce el número de horas de docencia directa presencial obligatoria a aquellos académicos que desarrollen determinadas funciones. A las "descargas" horarias tienen derecho los académicos que desempeñan cargos en la administración central de la universidad y quienes desarrollan otras tareas de interés institucional. En ambos casos las "descargas" no son sumativas y establecen la obligatoriedad mínima de un curso, en caso de que éstas fueran mayores a la obligación de docencia directa presencial (tablas 2 y 3 ). 
80 COMPROMISOS DE DESEMPEÑO, INCENTIVOS Y EVALUACIÓN DEL TRABAJO ACADÉMICO - Enrique Fernández D.

Tabla 2. Descargas horarias por tareas de administración

\begin{tabular}{|l|c|c|}
\hline \multirow{2}{*}{ Actividad } & \multicolumn{2}{|c|}{ Descarga horaria } \\
\cline { 2 - 3 } & Hrs. pedagógicas & Módulos \\
\hline Rector & 8 & 4 \\
\hline Vicerrector & 8 & 4 \\
\hline Decano & 6 & 3 \\
\hline Director de escuela o centro & 6 & 3 \\
\hline Director administrativo & 6 & 3 \\
\hline Secretario académico & 4 & 2 \\
\hline Jefes de departamento & 4 & 2 \\
\hline Jefes o coordinadores de posgrado & 4 & 2 \\
\hline Jefes o coordinadores de carrera & 4 & 2 \\
\hline
\end{tabular}

Tabla 3. Descargas horarias por tareas de interés institucional

\begin{tabular}{|l|c|c|}
\hline \multirow{2}{*}{ Actividad } & \multicolumn{2}{|c|}{ Descarga horaria } \\
\cline { 2 - 3 } & $\begin{array}{c}\text { Horas } \\
\text { pedagógicas }\end{array}$ & Módulos \\
\hline $\begin{array}{l}\text { Investigador responsable en proyectos Fondecyt, Fondef o } \\
\text { equivalentes }\end{array}$ & 6 & 3 \\
\hline $\begin{array}{l}\text { Coinvestigador en proyectos Fondecyt, Fondef o } \\
\text { equivalentes }\end{array}$ & 4 & 2 \\
\hline Director en proyectos Mecesup & 4 & 2 \\
\hline Director alterno en proyectos Mecesup & 4 & 2 \\
\hline Investigador responsable en proyectos PID* & 4 & 2 \\
\hline Coinvestigador en proyectos PID & 4 & 2 \\
\hline Investigador responsable en proyectos DIN** & 2 & 1 \\
\hline Coinvestigador en proyectos DIN & 2 & 1 \\
\hline Director de proyectos FAD*** & 2 & 1 \\
\hline Coinvestigador en proyectos FAD & 2 & 1 \\
\hline
\end{tabular}

* Proyectos internos de investigación de carácter interdisciplinario

** Proyectos internos de investigación

*** Proyecto de desarrollo de material o estrategias docentes

c) Modalidades de asignación de los incentivos monetarios

Existen básicamente dos modalidades: al individuo o a la unidad académica a la que está adscrito. La mayor complejidad que tiene la primera, a juicio de muchos, es que privilegia los desarrollos individuales por sobre los de las unidades como un todo. Además, al bonificar sólo determinadas actividades de interés institucional, se instala una sensación de injusticia entre quienes no rinden (por las 
razones que sea) en las actividades bonificadas, lo que lleva a algunos académicos a orientar su trabajo hacia determinadas tareas (para las cuales en ocasiones no están capacitados) en un intento por lograr bonificaciones económicas, abandonando otras igualmente relevantes para el funcionamiento de la universidad.

Algunas instituciones han privilegiado entregar los incentivos a la unidad a la que pertenecen los académicos y no pueden constituir remuneración. En este caso, pasan a formar parte del presupuesto de la unidad y su uso se negocia entre los propios académicos que la constituyen. Quienes defienden esta modalidad señalan que ella representa mejor el sentido de comunidad académica que debiera primar en las universidades, al privilegiar un desarrollo de conjunto por sobre el individual. Es decir, se reducen los niveles de injusticia del modelo anterior, ya que no todos los individuos tienen los mismos intereses, capacidades, ni talentos, pero, al tener colegas dedicados a otras actividades que no cuentan con incentivos (como extensión o administración, por ejemplo), algunos se pueden dedicar de lleno a las que sí cuentan con ellos (en general, investigación y publicaciones).

Sus detractores, en tanto, señalan que esta forma de asignación es injusta, ya que, si el grado de formalización del manejo presupuestario es bajo y, por lo mismo, quien dirige la unidad hace uso discrecional de los recursos y los académicos deben negociar cada vez determinados financiamientos (que conciben como propios) para sus actividades; por otra parte, con este modelo se "subvenciona" a quienes producen menos, de acuerdo con los estándares organizacionales.

Esta no es una disyuntiva fácil de resolver, ya que la aplicación de uno u otro modelo no se vincula sólo con estilos de gestión, sino también con filosofías organizacionales e, incluso, con conceptos de sociedad.

Por otra parte, los incentivos monetarios no son los únicos determinantes de la productividad académica, existe otra gran variedad 
de factores que incide en ello, como el nivel de perfeccionamiento de la planta de profesores, la motivación de ascender en las jerarquías académicas, los recursos propios invertidos en investigación, los sistemas de evaluación y calificación académica, entre otros.

El siguiente contrapunto puede ilustrar esta disyuntiva. La Pontificia Universidad Católica de Valparaíso y la Universidad de Talca tienen estructuras de incentivos bastante similares, pero modalidades de asignación distinta. Mientras la primera entrega los beneficios al académico, la segunda lo hace a la unidad en que éste trabaja (tabla 4).

Tabla 4. Productividad científica PUCV - UTAL, 2006

\begin{tabular}{|l|c|c|c|c|}
\cline { 2 - 5 } \multicolumn{1}{c|}{} & \multicolumn{2}{c|}{ PUC de Valparaíso } & \multicolumn{2}{c|}{ Universidad de Talca } \\
\hline Docentes jornada completa (djc) & \multicolumn{2}{|c|}{358} & $\mathrm{n}$ & $\mathrm{djc} / \mathrm{n}$ \\
\hline Actividad & $\mathrm{n}$ & $\mathrm{djc} / \mathrm{n}$ & $\mathrm{n}$ & 34,0 \\
\hline $\begin{array}{l}\text { Proyectos FONDECYT aprobados } \\
(2006)\end{array}$ & 21 & 17,0 & 6 & 0 \\
\hline $\begin{array}{l}\text { Proyectos FONDECYT de } \\
\text { posdoctorado (2006) }\end{array}$ & 2 & 179,0 & 0 & 204,0 \\
\hline $\begin{array}{l}\text { Proyectos FONDECYT de incentivo } \\
\text { a la cooperación internacional } \\
(2006)\end{array}$ & 4 & 89,5 & 1 & 8,9 \\
\hline $\begin{array}{l}\text { Proyectos FONDECYT en ejecución } \\
\text { (2006) }\end{array}$ & 42 & 8,5 & 23 & 2,8 \\
\hline Publicaciones ISI (2005) & 69 & 5,2 & 73 & \\
\hline
\end{tabular}

Fuente: INDICES 2007, CSE.

En la tabla 4 se puede apreciar que, tanto en términos de volumen (n) como proporcionalmente ( $\mathrm{djc} / \mathrm{n}$ ), los valores se comportan de manera muy disímil. Mientras la Pontificia Universidad Católica de Valparaíso es más eficiente en algunos indicadores, en otros lo es la Universidad de Talca. Es decir, con estructuras de incentivos similares pero con modalidades de asignación divergentes no se generan desarrollos marcadamente distintos. 


\section{Evaluación}

El funcionamiento óptimo de un instrumento como el compromiso de desempeño debe ir acompañado de un sistema de evaluación que verifique el grado de logro alcanzado y establezca sanciones para los incumplimientos reiterados.

Esto tiene, en general, dos perspectivas. La primera es de carácter netamente administrativa y se refiere a la verificación que hace el Departamento de Recursos Humanos y la Dirección de Docencia, o sus equivalentes, del cumplimiento de las obligaciones contractuales mínimas de cada profesor (por ejemplo, la realización de las horas mínimas de docencia). Evidentemente, no es este aspecto el que interesa en este artículo.

La segunda se refiere a una evaluación cualitativa del desempeño académico hecha por los pares. Algunas universidades realizan evaluaciones periódicas de sus profesores a través de comisiones, y éstas utilizan como uno de los insumos el grado de cumplimiento de los compromisos de desempeño (otros son las evaluaciones docentes que realizan los estudiantes, informes de los superiores, entre otros). A su vez, los resultados de estas comisiones son utilizados como referencia por las comisiones de categorización y promoción, que ponderan la "carrera" de los académicos.

En varios casos, además, los profesores deben realizar una autoevaluación del cumplimiento de su compromiso y en ella pueden especificar las causas que expliquen y justifiquen determinados incumplimientos, y los nuevos logros no considerados en el compromiso original que se deban adicionar o que sustituyen los no alcanzados.

La operatoria temporal de este proceso se presenta en el diagrama 2. 
Diagrama 2. Operatoria temporal del compromiso de desempeño académico y su evaluación

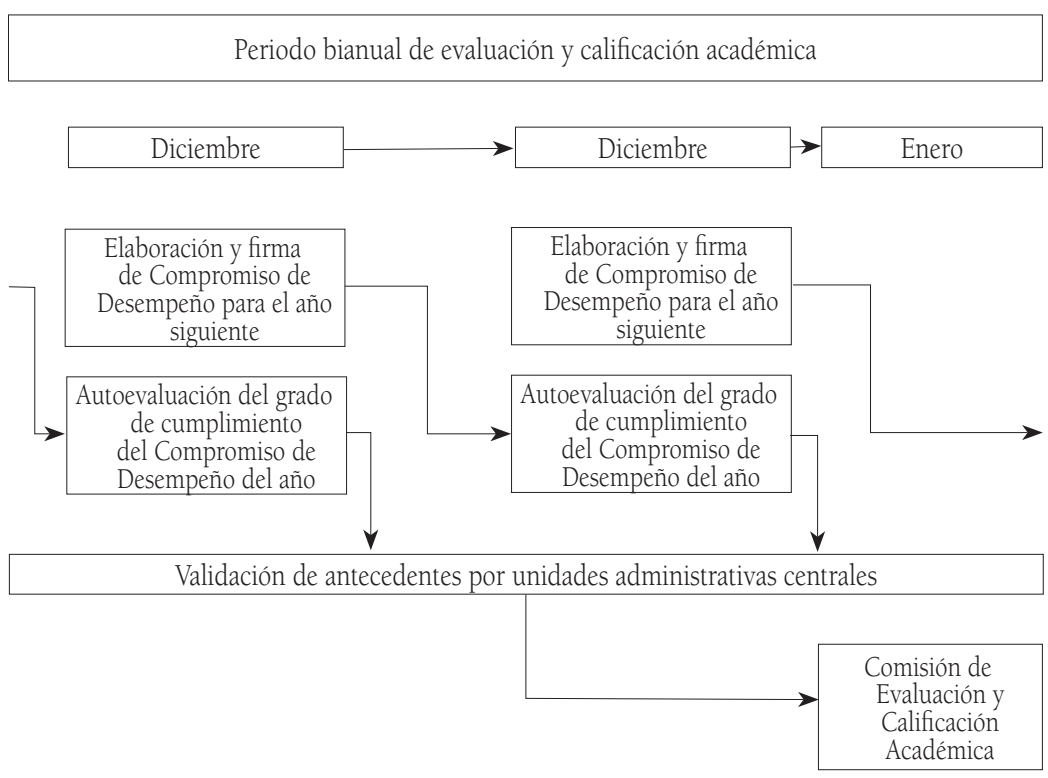

Las consecuencias por el incumplimiento reiterado de los compromisos contraídos, derivadas de las perspectivas administrativa y académica de la evaluación, son distintas. Mientras en el primer caso puede implicar la desvinculación inmediata del académico, en el segundo puede significar, en el corto plazo, un bajo o nulo avance en la carrera académica y, recién en el largo, una desvinculación de la universidad ${ }^{6}$.

\section{Compromisos, incentivos, evaluación y planificación estratégica}

Como se señaló, tanto los compromisos de desempeño como los incentivos al trabajo académico son instrumentos operativos de la planificación estratégica y cumplen la doble función de socializar

6 Salvo en aquellas universidades en que las evaluaciones por comisiones de pares tienen consecuencias administrativas. En este caso, el incumplimiento reiterado puede significar la desvinculación inmediata del académico. 
los objetivos de desarrollo al interior de la organización y servir de instrumentos para su logro. Por lo mismo, es imprescindible que estén alineados con ella y cuenten, además, con mecanismos evaluativos ${ }^{7}$.

En el diagrama 3 se presenta un esquema señalando el lugar que corresponde en la planificación estratégica a los compromisos de desempeño, los incentivos y la evaluación.

Diagrama 3. Compromisos de desempeño, incentivos y evaluación en el marco de la planificación estratégica

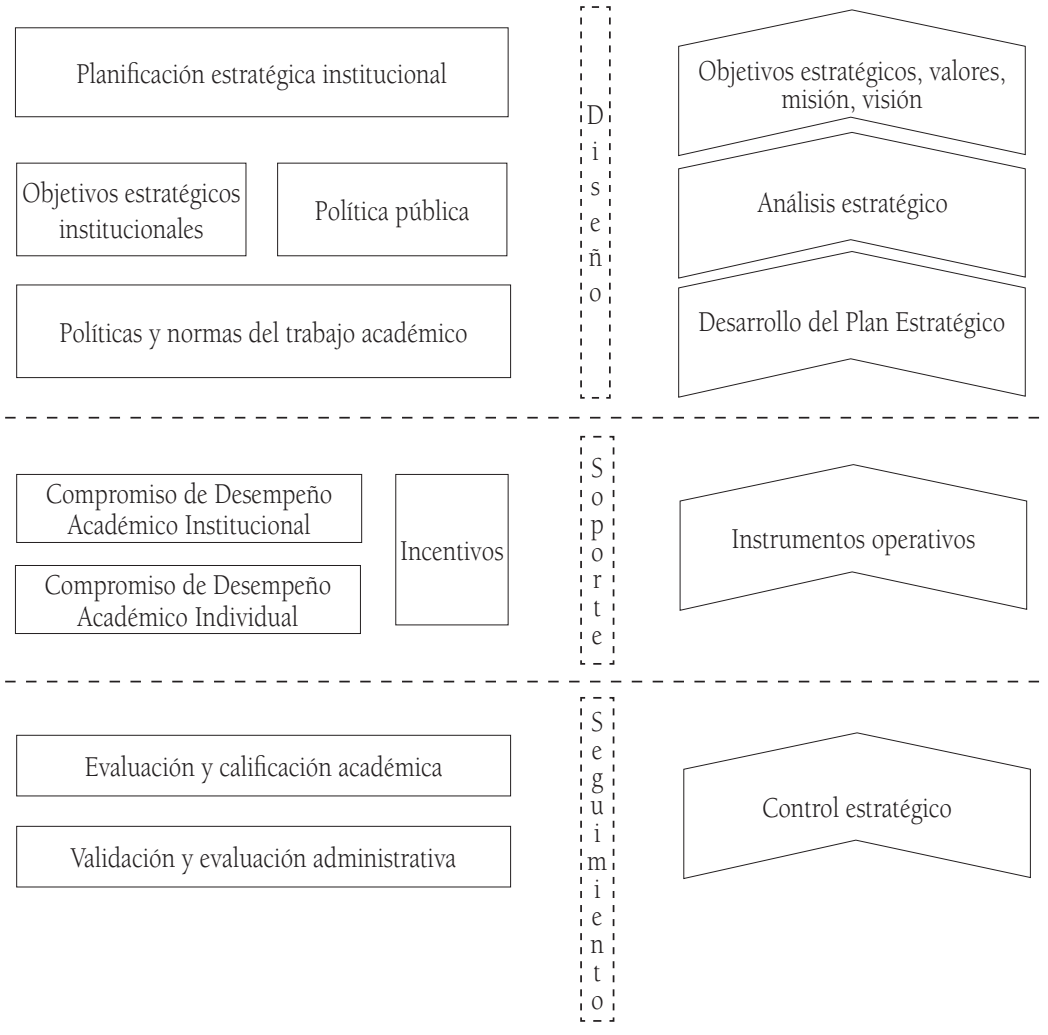

7 Una de las debilidades detectadas en las planificaciones estratégicas institucionales es, precisamente, la ausencia de instrumentos apropiados para desarrollar los planes de acción (Cáceres, 2007: 131). 


\section{Complejidades en la implementación de un compromiso de desempeño académico}

La implementación de compromisos de desempeño académico como un instrumento para el logro de los objetivos estratégicos institucionales es una tarea compleja y larga, al menos por la ausencia de una cultura organizacional en las universidades que reconozca legitimidad a estos instrumentos, la confluencia en estos procedimientos de dos lógicas profesionales habitualmente tensionadas en las universidades -la que proviene de la profesión académica y la que se origina en la gestión- y por la necesidad de consensuar los aspectos centrales de estos instrumentos.

En las universidades chilenas estos instrumentos se han aplicado en los últimos años y sólo en algunas de ellas. Incluso, en muchas no existen modelos de carrera académica que impliquen evaluaciones y calificaciones sistemáticas de los profesores (Atria, 2006: 85-106). Esto lleva a que los compromisos de desempeño sean percibidos como algo externo y no se les reconozca la legitimidad necesaria para coordinar el trabajo académico.

Esta situación se enlaza con el hecho de que en las universidades, cada vez más, se está haciendo patente la confluencia de dos racionalidades que han comenzado a colisionar: la lógica de la profesión académica y la lógica de la profesión administrativa. Académicos y gestores se ven enfrentados no sólo en los conceptos generales de universidad, sino también en la visión acerca de cómo se deben alcanzar los objetivos. Mientras los segundos ponen el énfasis en la escasez de los recursos y en la necesidad de cuantificar el trabajo y productos académicos, los primeros enfatizan su pertenencia a una comunidad científica que va más allá de su propia organización y que tiene sus propias normas de evaluación de calidad y productividad. Esto último provoca, como señalan muchos gestores, que no se perciba adecuadamente la condición de miembro de una organización específica y no pocas veces se pierda el sentido de responsabilidad para con ella. A la inversa, los profesores acusan con frecuencia a la 
administración de tener escaso conocimiento y comprensión de la lógica del mundo académico (Fernández y Stock, 2007).

Desde este punto de vista, parece evidente que una implementación exitosa de los compromisos de desempeño sólo es posible si se logra acuerdo entre gestores y académicos. Esto, sin embargo, requiere de un largo tiempo dedicado a las discusiones y diseño de los mismos y, por ello, como se señaló, su implementación se hace lenta.

Un último punto que parece necesario mencionar es que, a juicio de algunos entrevistados, un elemento central para el éxito de los compromisos de desempeño debe ser su simpleza y que no intenten llegar mediante escalas rígidas de puntuación a valores exactos de desempeño o, a través de asignaciones horarias, a excesivas precisiones de tiempos de trabajo. Esto conduciría sólo a una "falsa aritmética", es decir, a hacer ejercicios contables destinados a "cuadrar" el compromiso más que a intentar definir desempeños académicos de calidad y evaluables por los pares.

\section{Conclusiones}

Los compromisos de desempeño y los incentivos al trabajo académico son algunos de los instrumentos que han comenzado a ganar espacio en la gestión universitaria chilena. En ciertas instituciones ya se utilizan hace varios años con resultados diversos, en otras se encuentran en la fase de discusión, diseño o implementación ${ }^{8}$.

Para que alcancen un carácter sistémico, deben implementarse en el marco de una planificación estratégica, estar acordes con las políticas y normas del trabajo académico y tender a orientar el trabajo de los profesores en dirección del cumplimiento de los objetivos institucionales. Además, deben ir acompañados de un sistema de evaluación que verifique el grado de logro de los compromisos contraídos y sancione los incumplimientos reiterados. La ausencia de

8 Universidad de La Frontera y Universidad Austral de Chile. 
evaluación reduce en el corto plazo la legitimidad de los instrumentos $y$, por lo mismo, su uso se transforma en un ritual incapaz de coordinar el trabajo académico.

Dos elementos parecieran ser centrales en el éxito de algunas experiencias. Por una parte, su baja complejidad aritmética; por otra, que su diseño e implementación se realice en el marco de una discusión entre gestores y académicos, tendiente a consensuar sus aspectos centrales.

Como se señaló al comienzo el objetivo de este artículo no es prescribir el uso de estos instrumentos ni, mucho menos, augurar que a través de ellos se puede lograr una gestión eficiente. Sólo se busca presentar algunas experiencias y problematizar ciertos aspectos generales de su utilización. En efecto, queda prácticamente sin mencionar una de sus dimensiones más complejas: la gestión política de su implementación.

Este último aspecto tiene, en el caso de las universidades y producto de su especificidad organizacional, particular relevancia.

\section{Referencias bibliográficas}

Atria, Raúl (2006) La Gestión de las Universidades del Consejo de Rectores. Calidad en la Educación, 24, pp. 67-116.

Bernasconi, Andrés (2003) Organizational diversity in Chilean higher education: Faculty regimes in private and public universities. Dissertation: Boston University.

Bernasconi, Andrés y Rojas, Fernando (2004) Informe sobre la Educación Superior en Chile: 1980-2003. Santiago de Chile: Editorial Universitaria / IESALC.

Cáceres, Carlos (2007) Planeación Estratégica en Universidades del Consejo de Rectores. Evidencias del periodo 2000-2005. Calidad en la Educación, 27, pp.108-150.

Fernández, Enrique (2007) Los límites de la gestión: contradicciones entre la lógica organizacional y la lógica académica. Presentación en el Taller de Gestión de Educación Superior, Pucón, noviembre de 2007. 
Fernández, Enrique y Stock, Manfred (2007) Los límites de la gestión. Consideraciones sociológicas sobre la gestión universitaria en Chile y Alemania. Universum, 22(2), pp.108-124.

Kotler, Philip y Murphy, Patrick E. (1981) Strategic Planning for Higher Education. The Journal of Higher Education, 52 (5), pp. 470-489.

Montenegro, Enrique (2007) Algunos modelos para retribuir la calidad del desempeño y el compromiso institucional de los académicos en nuestras universidades. Presentación en el Taller de Gestión de Educación Superior, Pucón, noviembre de 2007.

Prieto, Juan Pablo (2007) Politicas, regulaciones e incentivos del trabajo académico en la Universidad de Talca. Presentación en el Taller de Gestión de Educación Superior, Pucón, noviembre de 2007.

Ziegele, Frank (2007a) Contract Management. Presentación en el Taller de Gestión de Educación Superior, Pucón, noviembre de 2007.

Ziegele, Frank (2007b) Strategic Management. Presentación en el Taller de Gestión de Educación Superior, Pucón, noviembre de 2007.

Entrevistas:

2003: Enrique Montenegro, vicerrector de Asuntos Docentes, Pontificia Universidad Católica de Valparaíso.

2003: Bernardo Domínguez, director general de Desarrollo Académico, y Luciano Gainza, responsable de Evaluación, Equipo de Desarrollo Académico, Pontificia Universidad Católica de Chile.

2003: Juan Antonio Rock, secretario general, Juan Pablo Prieto, vicerrector académico, y Patricio Ortúzar, director de Planificación y Análisis Institucional, Universidad de Talca.

Fuentes de información:

Programa de Mejoramiento de la Equidad y Calidad de la Educación Superior, www.mecesup.cl

Recibido: 2 de abril de 2008

Aprobado: 28 de mayo de 2008 